\title{
ПРОСТОЙ МЕТОД ОПРЕДЕЛЕНИЯ СОБСТВЕННЫХ ЧАСТОТ БИКОНИЧЕСКОГО РЕЗОНАТОРА
}

\author{
М. В. АНДРЕЕВ ${ }^{1}$, О. О. ДРОБАХИН ${ }^{1}$, Д. Ю. САЛТЫКОВ ${ }^{1}$, Н. Б. ГОРЕВ ${ }^{2}$, И. Ф. КОДЖЕСПИРОВА ${ }^{2}$ \\ ${ }^{1}$ Днепровский национальный университет им. О. Т. Гончара, \\ Украина, Днепр, 49050, ул. Научная, 13 \\ ${ }^{2}$ Институт технической механики НАН и НКА Украинь, \\ Украина, Днепр, 49600, ул. Ляшко-Попеля 15
}

\begin{abstract}
Аннотация. Ряд особенностей биконических резонаторов делает их привлекательными для различных применений. Выражения для вычисления собственных частот биконического резонатора с большими углами раскрыва конуса могут быть получены с использованием метода разделения перекрывающихся областей в сочетании с методом коллокации; однако выражения, приведенные в литературе, включают только одну пару точек коллокации, что не дает возможности оценить точность определения собственной частоты. Целью настоящей работы является вычисление собственных частот биконического резонатора для произвольного числа пар точек коллокации. Выведено уравнение для собственных частот биконического резонатора для азимутально-симметричного поперечного электрического поля при произвольном числе пар точек коллокации. Уравнение сводится к двум уравнениям, решение которых требует гораздо меньших вычислительных затрат по сравнению с исходным уравнением. В решениях одного из двух уравнений допускаются моды, симметричные относительно плоскости симметрии резонатора, а решения другого допускают антисимметричные моды. Вычисленные собственные частоты быстро сходятся с увеличением числа пар коллокационных точек, тогда как использование только одной пары точек коллокации может внести заметную ошибку. Предлагаемый метод может быть использован при разработке компонентов и узлов на основе биконических резонаторов.
\end{abstract}

Ключевые слова: биконический резонатор; метод коллокаций; дисперсионное уравнение; собственная частота; метод частично пересекающихся областей

\section{1. ВВЕДЕНИЕ}

Методы измерения на основе резонаторов традиционно используются для получения электрофизических параметров различных материалов $[1,2]$. Цилиндрические резонаторы, работающие на азимутальных симметричных модах, нашли широкое применение в качестве сенсоров [3-6]. Однако они имеют ряд недостатков: сдвиг резонансной частоты и уменьшение добротности, вызванные отверстиями для ввода образца в торцевые стенки, необходимость высокой степени ортогональности тор- цевых стенок относительно продольной оси и возможность трансформации режима работы в другой режим при внесении испытуемого образца в резонатор.

Биконические резонаторы в основном не имеют вышеуказанных недостатков. Из-за наличия зон затухания волн вблизи вершин конуca, здесь могут быть сделаны отверстия без существенного влияния на структуру поля или резонансные частоты [7]. Эта особенность позволяет использовать биконические резонаторы в различных инженерных задачах, например, при измерении смещения, при поточной 


\section{БИБЛИОГРАФИЧЕСКИЙ СПИСОК}

1. Egorov, V. N. Resonance methods for microwave studies of dielectrics (Review). Instruments and Experimental Techniques. Vol. 50, No. 2, P. 143-175, 2007. DOI: 10.1134/S0020441207020017.

2. Afsar, M. N.; Birch, J. R.; Clarke, R. N.; Chantry, G. W. The measurement of the properties of materials. Proc. IEEE, Vol. 74, No. 1, P. 183-199, 1986. DOI: 10.1109/PROC.1986.13432.

3. Bussey, H. E.; Morris, D.; Zal'tsman, E. B. International comparison of complex permittivity measurement at $9 \mathrm{GHz}$. IEEE Trans. Instrum. Meas., Vol. 23, No. 3, P. 235-239, 1974. DOI: 10.1109/TIM.19 74.4314270 .

4. Ni, E.; Stumper, U. Permittivity measurements using a frequency-tuned microwave TE01 cavity resonator. IEE Proc. H-Microwaves, Antennas and Propagation, Vol. 132, No. 1, P. 27-32, 1985. DOI: 10.1049/ip-h-2:198 $\underline{50005}$.

5. Kling, C. K.; Whites, K. W.; Groven, L. J. Accurate specimen placement for dielectric measurements in TM0n0 cylindrical cavity. Proc. of IEEE Int. Symp. on Antennas and Propagation, APSURSI, 26 June-1 July 2016, Fajardo, Puerto Rico. IEEE, 2016, P. 1995-1996, DOI: 10.1109/APS.2016.7696704.

6. Sydoruk, V. A.; Fiorani, F.; Jahnke, S.; Krause, H.-J. Design and characterization of microwave cavity resonators for noninvasive monitoring of plant water distribution. IEEE Trans. Microwave Theory Tech., Vol. 64, No. 9, P. 2894-2904, 2016. DOI: 10.1109/TMTT. 2016.2594218

7. О. О. Дробахин; П. И. Заболотный; Привалов, Е. Н. Учет влияния элементов связи на резонансные явления в биконических резонаторах. Известия вузов. Радиоэлектроника, Т. 53, № 7, C. 56-63, 2010. URI: http://radio.kpi.ua/article/view/S0021347010070071.

8. Drobakhin, O. O.; Privalov, Ye. N.; Saltykov, D. Yu. Open-ended waveguide cutoff resonators for monitoring dielectrics parameters of gases. Telecommun. Radio Eng., Vol. 72, No. 7, P. 627-640, 2013. DOI: 10.1615/TelecomRadEng.v72.i7.60.

9. Andreev, M. V.; Drobakhin, O. O.; Privalov, Ye. N.; Saltykov, D. Yu. Measurement of dielectric material properties using coupled biconical resonators. Telecommun. Radio Eng., Vol. 73, No. 11, P. 1017-1032, 2014. DOI: $10.1615 /$ TelecomRadEng.v73.i11.70.

10. Алимов, А. А.; Радионов, А. А. Расчет открытого предельного биконического резонатора. АнтенHbl, № 4, C. 40-45, 2015. URI: https://elibrary.ru/item. asp? $\mathrm{id}=23366163$.

11. Van’t Hof, J. P.; Stancil, D. D. Eigenfrequencies of a truncated conical resonator via the classical and Wentzel-Kramers-Brillouin methods. IEEE Trans. Microwave Theory Tech., Vol. 56, No. 8, P. 1909-1916, 2008. DOI: 10.1109/TMTT.2008.927408.

12. Kuryliak, D. B.; Nazarchuk, Z. T.; Trishchuk, B. O. Axially-symmetric TM-waves diffraction by sphere-conical cavity. PIER B, Vol. 73, P. 1-16, 2017. DOI: 10.2528/PIERB16120904.

13. Hoppe, R. H. W.; Kuznetsov, Yu. A. Overlapping domain decomposition methods with distributed Lagrange multipliers. J. Numerical Math., Vol. 9, No. 1, P. 285-293, 2001.

14. Дробахин, О. О.; Заболотный, П. И.; Привалов, Е. Н. К приближенному расчету собственных частот биконических микроволновых резонаторов. Известия вузов. Радиоэлектроника, Т. 56, № 3, С. 28-35, 2013. URI: http://radio.kpi.ua/article/view/S0021347013 $\underline{030035}$. 